\title{
Microhematocrito como índice de aclimatación a la altura, en residentes sanos no mineros del campamento cuprífero de Chuquicamata y nativos residentes en los Andes de Tarapacá y Antofagasta (Chile)
}

\section{Introducción}

El hematocrito se define como el porcentaje de un volumen de sangre total ocupado por los glóbulos rojos. Estos elementos figurados de la sangre poseen como función primordial contener el pigmento conocido como hemoglobina $(\mathrm{Hb})$, cuya misión fundamental, aunque no única, es transportar el oxígeno $\left(\mathrm{O}_{2}\right)$ desde la interfase atmósfera/sistema alveolocapilar pulmonar hasta los tejidos, que lo requieren para mantener la vida. La máxima capacidad de transporte de $\mathrm{O}_{2}$ por $1 \mathrm{gr}$ de $\mathrm{Hb}$ es de 1.38 (Fenn y Rahn 1964) o 1.39 (West 1975) o $1.34 \mathrm{ml}$ (Comroe et al. 1955; Comroe 1965). Los individuos jóvenes y sanos de nivel del mar poseen alrededor de $97.1 \%$ de la capacidad de su $\mathrm{Hb}$ unida al $\mathrm{O}_{2}$, valor que es conocido como saturación arterial de la $\mathrm{Hb}$. con $\mathrm{O}_{2}\left(\mathrm{SaO}_{2}\right.$ en \%) (Comroe et al. 1955).

El porcentaje de saturación depende fundamentalmente de la cantidad de $\mathrm{O}_{2}$ disuelto en la sangre y de la presión que éste ejerce. El valor de $97.1 \%$ se obtiene en sangre arterial a $\mathrm{pH}$ 7.40; presión arterial de anhídrido carbónico $\left(\mathrm{paCO}_{2}\right)$ de $41 \mathrm{mmHg}$; temperatura de $38^{\circ} \mathrm{C}$. y con una presión arterial de $\mathrm{O}_{2}\left(\mathrm{paO}_{2}\right)$ de 95 a $100 \mathrm{mmHg}$ (Comroe et al. 1955; Comroe 1965). La relación entre la $\mathrm{paO}_{2}$ (eje de las $\mathrm{x}$ ) y la $\mathrm{SaO}_{2}$ (eje de las y) dibuja una curva sigmoidea, en forma de $\mathrm{S}$, conocida como curva de Barcroft o de disociación de la oxihemoglobina (Barcroft 1914).

Sin embargo, la curva que dibuja la afinidad de la $\mathrm{Hb}$ por el $\mathrm{O}_{2}$, a distintos niveles de $\mathrm{pO}_{2}$ de la sangre que circunda el glóbulo rojo, no es estática. Ella se modifica por una serie de variables: el $\mathrm{pH}$; la $\mathrm{pCO}_{2}$; la temperatura; el contenido de fosfatos orgánicos en el glóbulo rojo, de preferencia el 2-3 DPG y en menor

1 Centro de Investigaciones Ecobiológicas y Médicas de Altura, Hospital Roy H. Glover, Chuquicamata, CHILE.

2 Servicio Anestesiología. Hospital Roy H. Glover, Chuquicamata, CHILE.

3 Hospital Roy H. Glover, Chuquicamata, CHILE.
Raimundo SAntolaya ${ }^{1}$, José Araya ${ }^{2}$ y RaÚl Prieto ${ }^{3}$

proporción ATP y, finalmente, la concentración de aniones y cationes inorgánicos. (Comroe et al. 1955; Dejours 1962; Rossier et al. 1962; Fenn y Rahn 1964; Comroe 1965; McConn y Derrick 1972; West 1975). Esta afinidad variable es fundamental en la fisiología permitiendo adecuarse para una óptima captación en el pulmón y una entrega suficiente en los tejidos, polos de muy distintas condiciones fisicoquímicas. La convierte además en la reguladora del aporte de $\mathrm{O}_{2}$ durante el ejercicio, las condiciones patológicas y la vida en atmósferas que como en la altura poseen baja presión inspirada de $\mathrm{O}_{2}\left(\mathrm{PIO}_{2}\right)$, otorgándole el rango de proceso adaptativo de primer orden en la entrega tisular de $\mathrm{O}_{2}$. Existen además numerosas hemoglobinas patológicas, de afinidad aumentada o disminuida que no serán tratadas aquí.

La cantidad de $\mathrm{O}_{2}$ transportada a los tejidos depende de:

1. La cantidad de Hb de la sangre: $152 \mathrm{gr} / \mathrm{lt}$ a nivel del mar.

2. La saturación arterial de la $\mathrm{HbO}_{2}: 98 \%$ a nivel del mar. Ello significa que si 1 gr posee una capacidad máxima de transporte de $1.34 \mathrm{mlO}_{2}$, a esa saturación movería $1.313 \mathrm{mlO}_{2}$ por gr y $199.6 \mathrm{ml}$ para los $152 \mathrm{gr}$ de cada lt.

3. Del débito cardíaco: es la cantidad de sangre que el corazón mueve en un minuto. Su valor a nivel del mar es de 5.98 lt.

Por lo tanto, a nivel del mar el transporte de $\mathrm{O}_{2}$ será de 5.98 por $199.6 \pm 1193.7 \mathrm{mlO}_{2}$ por minuto. Estos valores pertenecen a los trabajos de Rotta (1956) y Velásquez (1972).

En la altura, la baja $\mathrm{PIO}_{2}$, proporcional al nivel de altitud, origina una $\mathrm{paO}_{2}$ más baja. En Chuquicamata, a $2800 \mathrm{~m}$ es de $61.2 \mathrm{mmHg}$ en 176 hombres sanos no mineros residentes permanentes, de edades entre 21 y más de 66 años (D.S.: $41 \mathrm{mmHg}$ ) y de $62 \mathrm{mmHg}$ en 162 mujeres (D.S.: $4.5 \mathrm{mmHg}$ ) (Santolaya et 
al. 1981). En Morococha, a $4540 \mathrm{~m}$, en los Andes Centrales, es de $45.1 \mathrm{mmHg}$ en 32 hombres sanos (Velásquez 1972: 102). Las saturaciones medias de $\mathrm{HbO}_{2}$ son de 92 y $78.1 \%$, respectivamente.

Desde este único ángulo de mira, el transporte de $\mathrm{O}_{2}$ estaría disminuido si no se compensara con un incremento del débito cardíaco y/o de la cantidad de $\mathrm{Hb}$ o de su parámetro complementario, el hematocrito (Hto). Usando el mismo estudio de Rotta y colaboradores (1956), en Morococha en cuatro jóvenes nativos de 23.7 años de edad media, el débito cardíaco fue de $5.29 \mathrm{lt} / \mathrm{min}$, algo menor que en siete individuos de Lima (5.98 lt/min), por lo que el principal parámetro compensador fue el incremento de la $\mathrm{Hb}$, que en Morococha fue de 20.7 $\mathrm{gr} / 100 \mathrm{ml}$, con $\mathrm{SaO}_{2}$ de $79.6 \%$. Todo ello permite deducir un transporte de $\mathrm{O}_{2}$ a la periferia de: $207 \mathrm{gr}$ $\mathrm{Hb} / \mathrm{litro} \times 1.34$ × $79.6=106.7$ × $5.29=1168 \mathrm{ml} / \mathrm{O}_{2} /$ min, cifra mayor que la transportada en Lima con 152 gr de $\mathrm{Hb}$ a $98 \%$ de saturación.

Desde los primeros estudios realizados por Viault (1891) en nativos de altura en Morococha y en visitantes prolongados a la altitud, se comprobó el incremento del número de glóbulos rojos en la sangre (Viault 1891; Mayorga 1978). Lo mismo demuestra Barcroft (1925), en su célebre expedición a Cerro de Pasco, quien describe en la Oroya (Perú), a $3900 \mathrm{~m}$ la presencia en todos los nativos de mayor número de glóbulos rojos y de cantidad de $\mathrm{Hb}$, aunque de cuantía variable. Monge (1929) ratifica estos hallazgos y describe la enfermedad que hoy lleva su nombre, por desaclimatación a la altura en nativos, cuyos portadores sufrían de una "eritremia" superior a la media del lugar.

Hurtado introduce técnicas fisiológicas más finas en el estudio, entre ellas, el Hto y la función arterial, lo que le permite ratificar lo anterior y publicar sus investigaciones mostrando una estrecha correlación entre nivel de altitud, descenso de la $\mathrm{SaO}_{2}$ e incremento de la $\mathrm{Hb}$ (Hurtado et al. 1945; Hurtado 1964). También en 1935 tiene lugar la expedición de la Universidad de Harvard a la región de Antofagasta encabezada por Keys (Keys et al. 1936), cuyos datos aparecidos en diversas publicaciones ratifican lo anterior. Dill y colaboradores $(1931,1936)$ correlacionan bien $\mathrm{la}_{\mathrm{paO}}$ y el porcentaje de $\mathrm{SaO}_{2}$ en una curva de Barcroft, con valores obtenidos en diversas alturas, entre ellas
Chuquicamata, Ollagüe (3660 m); Montt (4700 m) y Aucalquincha (5340 m), todos asentamientos de la región chilena en que hoy trabajamos.

Monge (1964, Monge et al. 1965) describen la ecuación que correlaciona estos parámetros que, desde entonces, adquieren carácter de ley de la aclimatación a la altura. En 83 sujetos de Morococha se fija el Hto medio en $59.5 \%$ a diferencia del hallado en Lima, de 46.6\% (Merino 1950; Hurtado et al. 1956a y b; Velásquez 1972).

A lo anterior se añaden hasta ahora innumerables trabajos que muestran el incremento de la actividad eritropoyética en la altura; del metabolismo del hierro; de la destrucción hemática; del rol de la eritropoyetina en esta respuesta, etc.

El presente trabajo describe los resultados de microhematocrito obtenidos en diversas comunidades humanas residentes a diferentes altitudes de los Andes del norte de Chile, que se diferencian además en su estilo de vida.

\section{Material y métodos}

Los hematocritos cuyos resultados se presentan fueron practicados a poblaciones residentes en ecosistemas $\mathrm{y}$ asentamientos diversos ubicados en las regiones de Tarapacá y Antofagasta durante los últimos 13 años. Estos grupos humanos se diferencian, no sólo por las características de su hábitat, sino por su definición racial, su permanencia individual, lo ancestral en la altura, su estilo de vida y tipo de asentamiento, sus hábitos, nivel de sedentarismo y grado de contaminación ambiental. En este trabajo se exponen los resultados de tres grupos:

\section{Campamento minero de Chuquicamata}

Comprende 271 hombres y 267 mujeres sanas, nativas o no de la altura, residentes por más de dos años en el campamento, sin antecedentes de trabajo en minería (fundición y molienda). El campamento se encuentra ubicado a $2800 \mathrm{~m}$ de altura sobre el nivel del mar $\left(\mathrm{P}_{\mathrm{B}}=548 \mathrm{mmHg}\right)$, en el desierto marginal de altura de la Provincia de El Loa, Región de Antofagasta, a $22^{\circ} 15^{\prime}$ S y $68^{\circ} 55^{\prime} \mathrm{O}$. Se trata de una población heterogénea desde el punto de vista racial, con predominio mestizo; con hábitos de vida occidentales, sedentarismo predominante, obesidad $\mathrm{y}$ tabaquismo frecuentes y niveles altos y variables de contaminación atmosférica. Las migraciones a 
la costa son habituales, siendo más asiduas en los estratos socioeconómicos más altos. El hematocrito fue estudiado en el marco de un complejo protocolo en marcha desde junio de 1977 que comprende: datos personales - anamnesis personal remota y actual - examen físico completo y exámenes complementarios que incluyen radiografía frontal y lateral de tórax - electrocardiograma de reposo - estudio funcional respiratorio - gases y equilibrio ácido base en sangre arterial y parámetros bioquímicos venosos: hemoglobina - micro y macrohematocrito - glicemia - colesterol - triglicéridos. Los datos aquí presentados abarcan hasta julio de 1982.

\section{Grupo Isluga}

Comprende seis hombres y seis mujeres, todos sanos nativos y residentes permanentes de los pueblos ancestrales de la ribera del río bofedal de Isluga que transcurre por el altiplano chileno de la Región de Tarapacá y desemboca en el Salar de Coipasa. El grupo es étnicamente homogéneo, constituido por indígenas que hablan aymara y aun el puquina; habitan en pequeños villorrios de adobe y paja brava $(i c h u)$; mantienen una economía de sustento basada en la agricultura y ganadería, que les obliga a una condición seminómade. No conocen el mar ni descienden de la altura del altiplano. Obesidad nula, hábitos tradicionales protegidos de la occidentalización gracias a la cordillera occidental andina; no fumadores, están sometidos a intensa actividad física, que les lleva en busca de leña hasta el borde mismo de las nieves eternas. No existe contaminación atmosférica. Los pueblos se asientan entre 4100 y $3650 \mathrm{~m}$ de altura. El protocolo comprendió, además del hematocrito, anamnesis - examen físico - electrocardiograma de reposo - estudio funcional respetarlo - PPD - y, en un grupo menor, evaluación de la capacidad aeróbica mediante bicicleta ergómetro. Los exámenes fueron realizados en un laboratorio base instalado en tres ocasiones, entre los años 1969 y 1971, en la hacienda Cusa Llapu, de CORFO, ubicada a $3650 \mathrm{~m}$, en el pueblo de Colchane, provincia entonces de Iquique, Región de Tarapacá, a $19^{\circ} 15^{\prime}$ 'S y $68^{\circ} 48^{\prime} \mathrm{O}$, con $\mathrm{P}_{\mathrm{B}}$ $\mathrm{m}=484 \mathrm{mmHg}$. Los hematocritos fueron realizados allí y en los pueblos de Isluga y Enquelga, vecinos a los $4000 \mathrm{~m}$ de altura.

\section{Grupo Socaire}

Comprende 21 hombres y 54 mujeres sanas, nativas y residentes permanentes del pueblo Licanantay o atacameño de Socaire, ubicado a $3450 \mathrm{~m}$ de altura
$\left(\mathrm{P}_{\mathrm{B}} \mathrm{m}=517 \mathrm{mmHg}\right)$, en las faldas occidentales de la cordillera occidental andina de la Región de Antofagasta, verdadero balcón sobre el Salar de Atacama, hoya endorreica de drenaje de las aguas de esa región andina. El pueblo se encuentra ligeramente al sur del trópico de Capricornio, a 2336' Lat S y $68^{\circ} 6^{\prime}$ Long $\mathrm{O}$. El grupo es étnicamente homogéneo. Sus nativos viven de la agricultura y ganadería. Son escasamente nómades y su actividad física es menos rigurosa que la de Isluga. No descienden a nivel del mar pero lo hacen con frecuencia a tierras más bajas del Salar, a $2400 \mathrm{~m}$. Mantienen un estilo de vida poco influido por los hábitos occidentales, siendo el tabaquismo y la obesidad escasas y la contaminación atmosférica, nula.

Los microhematocritos de Chuquicamata fueron realizados a través de punción venosa, depositando en un portaobjeto dos gotas de la sangre recién extraída. Con ella se cargaron, por aspiración pasiva, dos tubos capilares heparinizados de $75 \mathrm{~mm}$, marca Red Tube Sherwood Red Tip. Se sellaron con plasticina y se centrifugaron de inmediato durante cuatro minutos a 11000 rpm, en una centrífuga Janetzki THIZ. Toda la investigación fue realizada por el autor y sus colaboradores, ajenos a la rutina del Laboratorio Central del Hospital Roy H. Glover, donde tiene su asiento el Centro de Investigaciones Ecobiológicas y Médicas de Altura. Los de Socaire fueron realizados por el autor, mediante punción por lanceta del dedo medio de la mano izquierda, suficiente para lograr un flujo de sangre espontáneo desde el cual se llenaron por aspiración pasiva los mismos capilares ya descritos. Se utilizó la misma centrífuga, periódicamente calibrada con estroboscopio. La experiencia de Isluga se practicó por igual procedimiento descrito en Socaire, utilizando una centrífuga microcapilar internacional, modelo MB. Se han presentado datos parciales de las tres experiencias, en distintos encuentros y publicaciones (Donoso et al. 1971, 1976; Santolaya et al. 1973, 1976, 1981, 1982, 1983; Santolaya 1975, 1981; Santolaya y Donoso 1976).

\section{Resultados}

El Gráfico 1 resume el número total de casos (737 microhematocritos) distribuidos en $73 \%$ de Chuquicamata (538 casos); $17 \%$ de Isluga (124 casos) y $10 \%$ de Socaire ( 75 casos). El Gráfico 2 expone en un histograma la muestra completa, distribuida según sexo: 383 mujeres (267 de Chuquicamata; 62 de Isluga y 


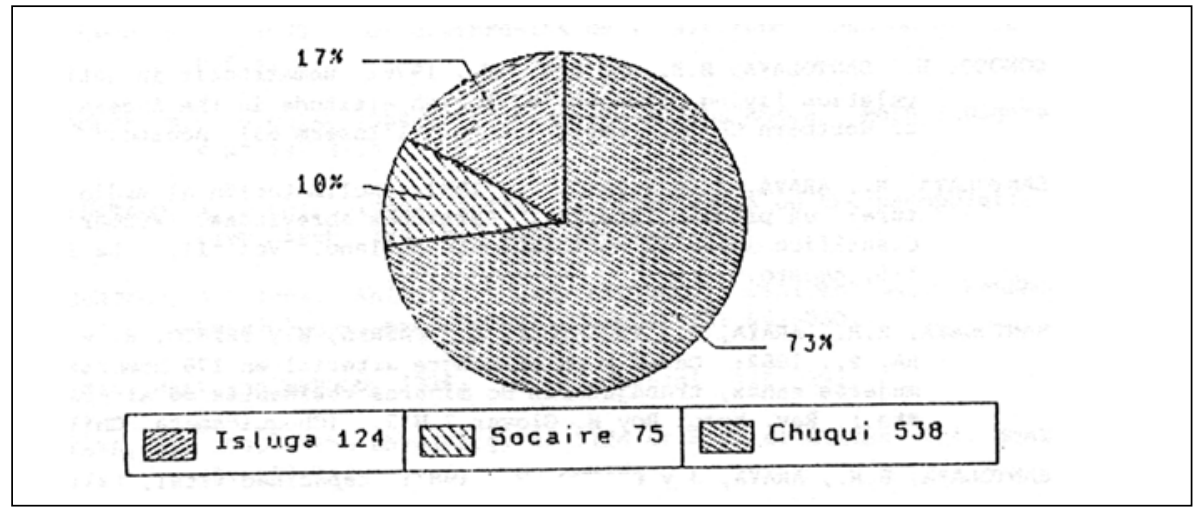

Gráfico 1. Distribución de 737 Micro-Hto de residentes de altura, sanos según localidades estudiadas. (Tarapacá y Antofagasta).

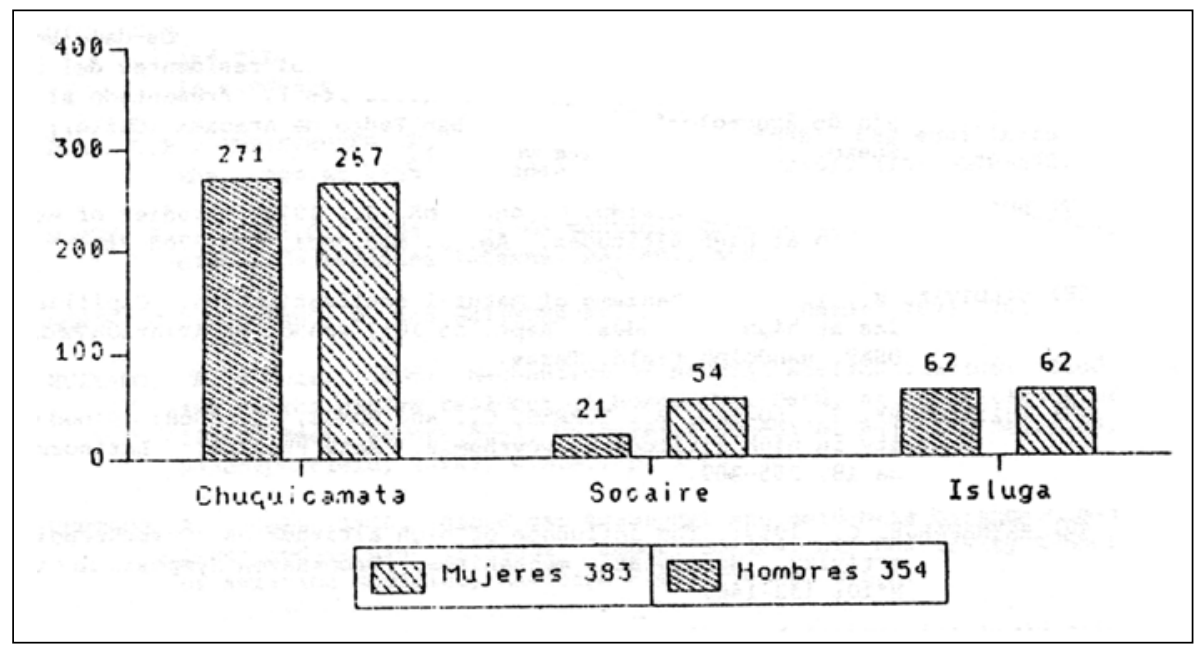

Gráfico 2. Distribución de 737 Micro-Hto de residentes de altura, sanos según sexo y localidades estudiadas. (Tarapacá y Antofagasta).

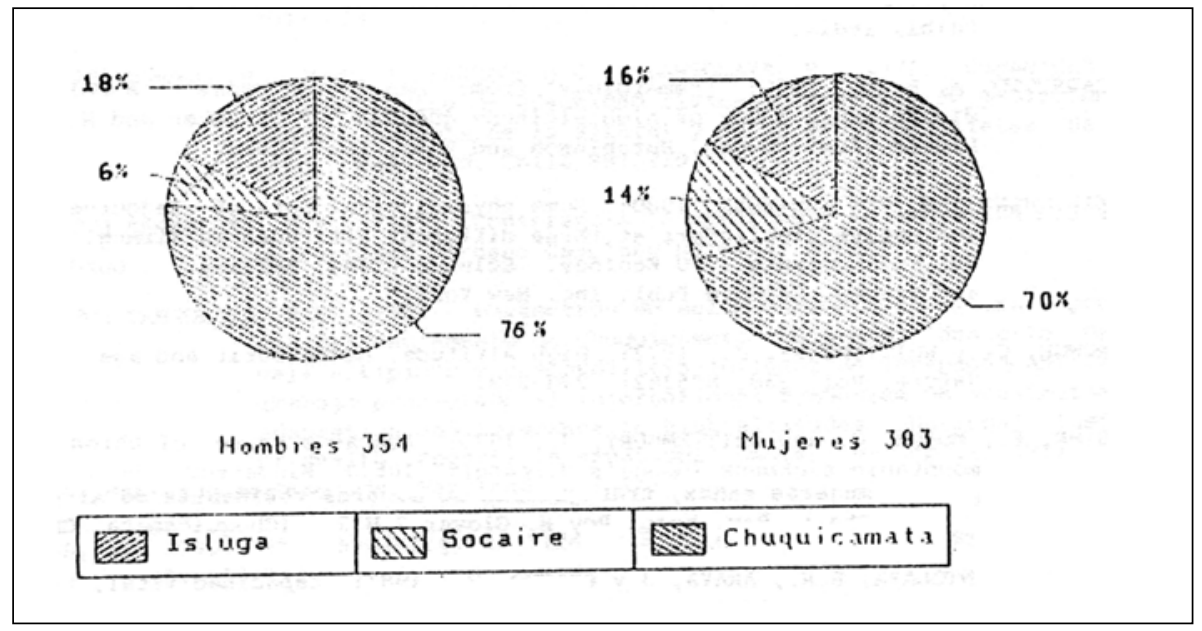

Gráfico 3. Distribución de 737 Micro-Hto de residentes de altura, sanos según sexo y localidades estudidadas. (Tarapacá y Antofagasta). 


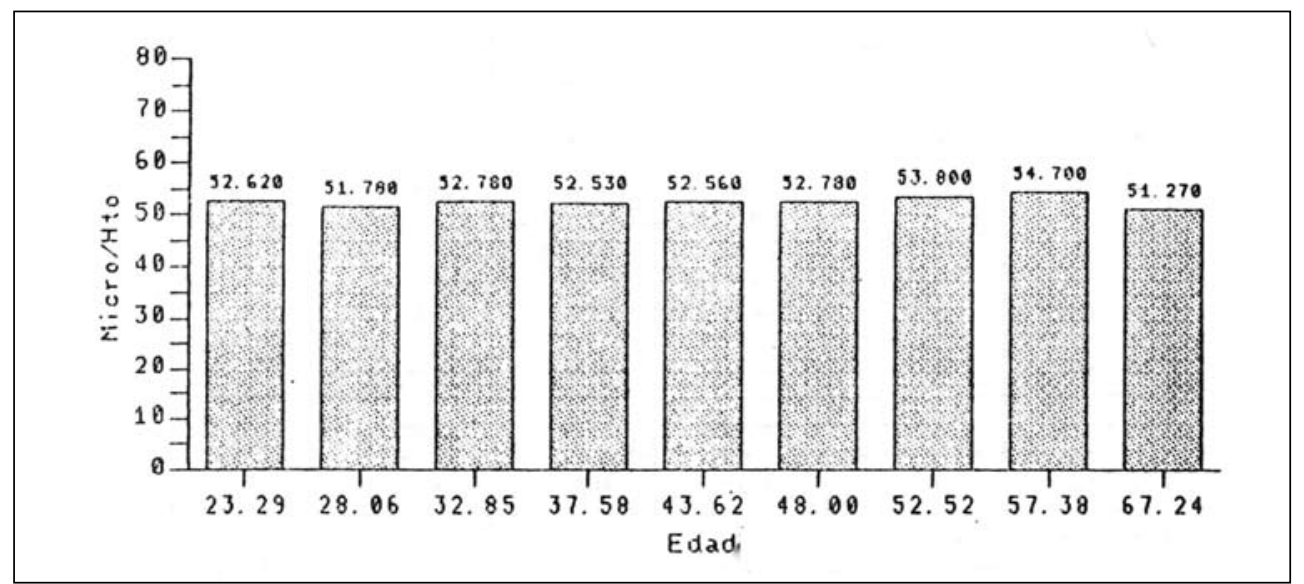

Gráfico 4. Micro-Hto en 271 hombres sanos residentes en campamento Chuquicamata (Chile), 2800 m.snm. Promedios según grupos de edad.

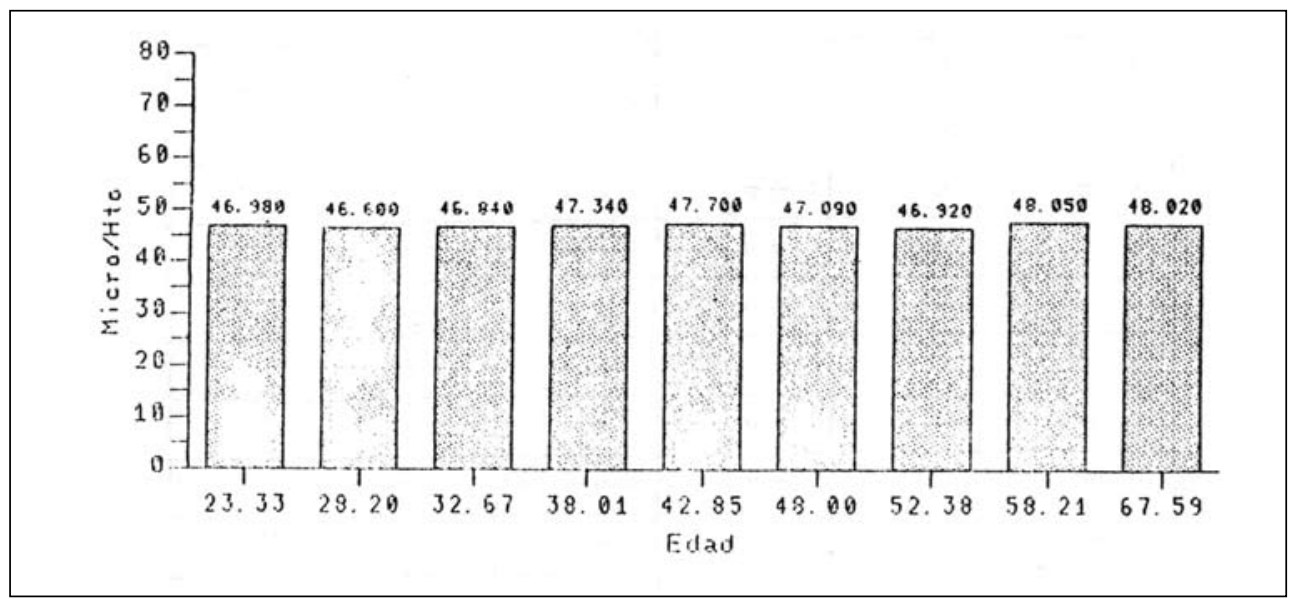

Gráfico 5. Micro-Hto en 267 mujeres sanas residentes en campamento Chuquicamata (Chile), 2800 m.snm. Promedios según grupos de edad.

54 de Socaire) y 354 hombres (271 de Chuquicamata; 62 de Isluga y 21 de Socaire). El Gráfico 3 muestra el porcentaje de hombres y mujeres de cada asentamiento estudiado, respecto del total.

Los Gráficos 4 y 5 muestran en forma de histograma las medias del microhematocrito para cada grupo etario quinquenal, entre 21 y más de 61 años, en Chuquicamata, para hombres y mujeres, respectivamente.

Los Gráficos 6 y 7 presentan, en forma de cuadro, el número de casos, la edad media, el hematocrito medio, la desviación estándar y el rango, para cada grupo etario quinquenal, en Chuquicamata, para los 271 hombres y 267 mujeres, respectivamente.
Los Gráficos 8 y 9 correlacionan el microhematocrito y la edad, en forma de nube de puntos, dibujándose la línea y valor de su coeficiente, en Chuquicamata, para hombres y mujeres, respectivamente. Los valores de 0.117 para la serie masculina y de 0.089 para la femenina, demuestran la ausencia de correlación entre ambos parámetros estudiados.

Los Gráficos 10 y 11 muestran en forma de histograma, las medias de los hematocritos obtenidos en Isluga, para hombres y mujeres respectivamente, según grupos etarios quinquenales, entre 11 y 20 años y decenales de 21 a más de 61 años.

Los Gráficos 12 y 13 presentan en forma de cuadro, el número de casos, la edad media, el hematocrito 
RAIMUNDO SANTOLAYA, JOSE ARAYA, RAUL PRIETO

\begin{tabular}{|c|c|c|c|c|c|}
\hline \multirow{2}{*}{ Edad (años) } & \multirow{2}{*}{$\mathbf{N}^{\circ}$ de casos } & \multirow{2}{*}{ Edad media } & \multicolumn{3}{|c|}{ Hematocrito } \\
\hline & & & Media & D.D. & Rango \\
\hline $21-25$ & 31 & 23.29 & 52.62 & 2.95 & $\begin{array}{l}46.3 \\
60.8\end{array}$ \\
\hline $26-30$ & 43 & 28.06 & 51.78 & 2.88 & $\begin{array}{l}46.5 \\
59.5\end{array}$ \\
\hline $31-33$ & 32 & 32.85 & 52.78 & 2.74 & $\begin{array}{l}48.8 \\
59.5\end{array}$ \\
\hline $36-40$ & 37 & 37.58 & 52.53 & 3.40 & $\begin{array}{l}46.3 \\
60.5\end{array}$ \\
\hline $41-45$ & 28 & 43.62 & 52.56 & 4.14 & $\begin{array}{l}45.5 \\
63.5\end{array}$ \\
\hline $46-50$ & 44 & 48.00 & 52.78 & 3.58 & $\begin{array}{l}47.0 \\
63.8\end{array}$ \\
\hline $51-55$ & 29 & 52.52 & 53.80 & 3.64 & $\begin{array}{l}46.5 \\
61.8\end{array}$ \\
\hline $56-60$ & 14 & 57.38 & 54.70 & 4.29 & $\begin{array}{l}46.0 \\
63.5\end{array}$ \\
\hline 61 y más & 13 & 67.24 & 51.2 & 74.42 & $\begin{array}{l}3.8 \\
60.0 \\
\end{array}$ \\
\hline Total & 271 & & 52.65 & 3.41 & $\begin{array}{l}43.8 \\
63.8\end{array}$ \\
\hline
\end{tabular}

Gráfico 6. Microhematocrito en 271 hombres sanos, trabajadores no mineros, residentes en campamento cuprífero de Chuquicamata, Chile. Altura: $2800 \mathrm{~m} . \mathrm{snm}-\mathrm{PBm}=548 \mathrm{mmHg}$. Distribución según edad.

\begin{tabular}{|c|c|c|c|c|c|}
\hline \multirow{2}{*}{ Edad (años) } & \multirow{2}{*}{$\mathbf{N}^{\circ}$ de casos } & \multirow{2}{*}{ Edad media } & \multicolumn{3}{|c|}{ Hematocrito } \\
\hline & & & Media & D.D. & Rango \\
\hline $20-25$ & 21 & 23.33 & 46.38 & 2.95 & $\begin{array}{l}46.3 \\
60.8\end{array}$ \\
\hline $26-30$ & 62 & 28.20 & 46.60 & 2.73 & $\begin{array}{l}41.0 \\
52.8\end{array}$ \\
\hline $31-35$ & 43 & 32.67 & 46.34 & 2.25 & $\begin{array}{l}43.8 \\
54.8\end{array}$ \\
\hline $36-40$ & 38 & 38.01 & 47.34 & 2.73 & $\begin{array}{l}41.0 \\
54.5\end{array}$ \\
\hline $41-45$ & 24 & 42.85 & 47.70 & 2.87 & $\begin{array}{l}41.5 \\
53.3\end{array}$ \\
\hline $46-50$ & 35 & 48.00 & 47.09 & 3.20 & $\begin{array}{l}40.8 \\
54.0\end{array}$ \\
\hline $51-55$ & 16 & 52.38 & 46.92 & 2.86 & $\begin{array}{l}42.3 \\
51.0\end{array}$ \\
\hline $56-60$ & 14 & 58.21 & 48.05 & 3.37 & $\begin{array}{l}42.8 \\
54.8\end{array}$ \\
\hline 61 y más & 13 & 67.59 & 46.02 & 2.16 & $\begin{array}{l}44.3 \\
52.8\end{array}$ \\
\hline Total & 267 & & 47.00 & 3.34 & $\begin{array}{l}40.5 \\
54.8\end{array}$ \\
\hline
\end{tabular}

Gráfico 7. Microhematocrito en 267 mujeres sanas, trabajadoras no mineras, residentes en campamento cuprífero de Chuquicamata, Chile. Altura: 2800 m.snm - PBm = 548 mmHg. Distribución según edad. 


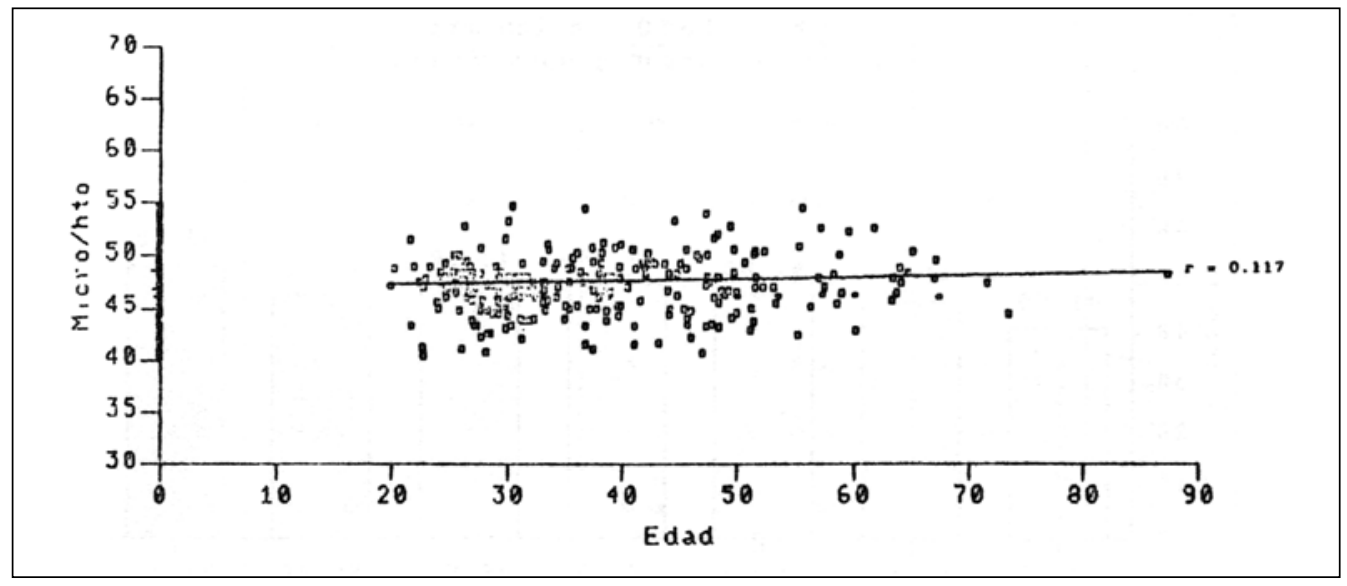

Gráfico 8. Micro-Hto en relación a edad en 267 mujeres trabajadoras sanas residentes en campamento Chuquicamata $(2800 \mathrm{~m})$.

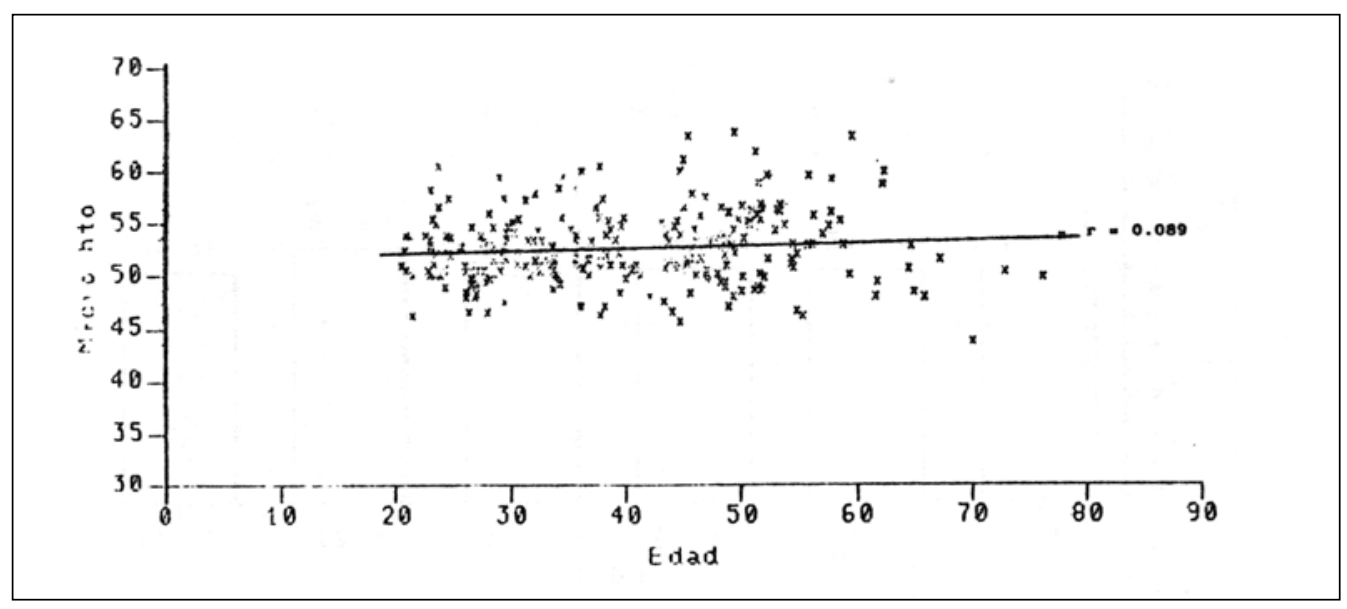

Gráfico 9. Micro-Hto en relación a edad en 271 hombres trabajadores no mineros residentes en campamento Chuquicamata $(2800 \mathrm{~m})$.

medio, la desviación estándar y el rango para cada grupo etario, en los 62 hombres y 62 mujeres de Isluga, respectivamente.

Los Gráficos 14 y 15 muestran el comportamiento de la correlación entre microhematocrito y edad, en los hombres y mujeres de Isluga, respectivamente, dibujando la línea y el valor de su coeficiente. No aparece en el gráfico el punto correspondiente a un hombre de edad probable 120 años, pero su valor fue incluido para el cálculo del coeficiente que fue de 0.52 para los hombres y 0.190 para las mujeres.

Los Gráficos 16 y 17 muestran en forma de histograma, las medias de los hematocritos obtenidos en
Socaire para hombres y mujeres, respectivamente, según grupos etarios decenales, a excepción del primer grupo entre 16 y 20 años de edad.

Los Gráficos 18 y 19 presentan en forma de cuadro el número de casos, la edad media, el hematocrito medio, la desviación estándar y el rango, para cada grupo etario, de los 21 hombres y 54 mujeres de Socaire, respectivamente.

Finalmente, en los Gráficos 20 y 21 se dibuja el comportamiento de la correlación entre microhematocrito y edad, en los hombres y mujeres de Socaire, respectivamente. Los coeficientes de correlación fueron 0.076 para la serie masculina y 0.17 para la femenina. 


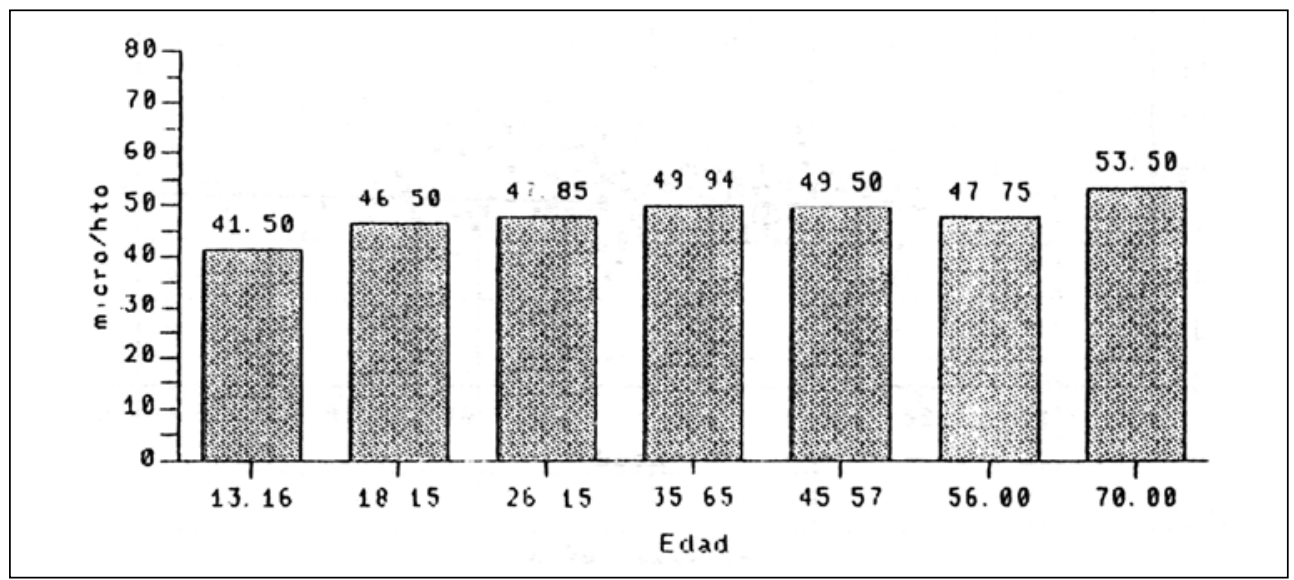

Gráfico 10. Micro-Hto en 62 hombres nativos sanos residentes de Isluga (3650-4100 m) promedios según grupos de edad.

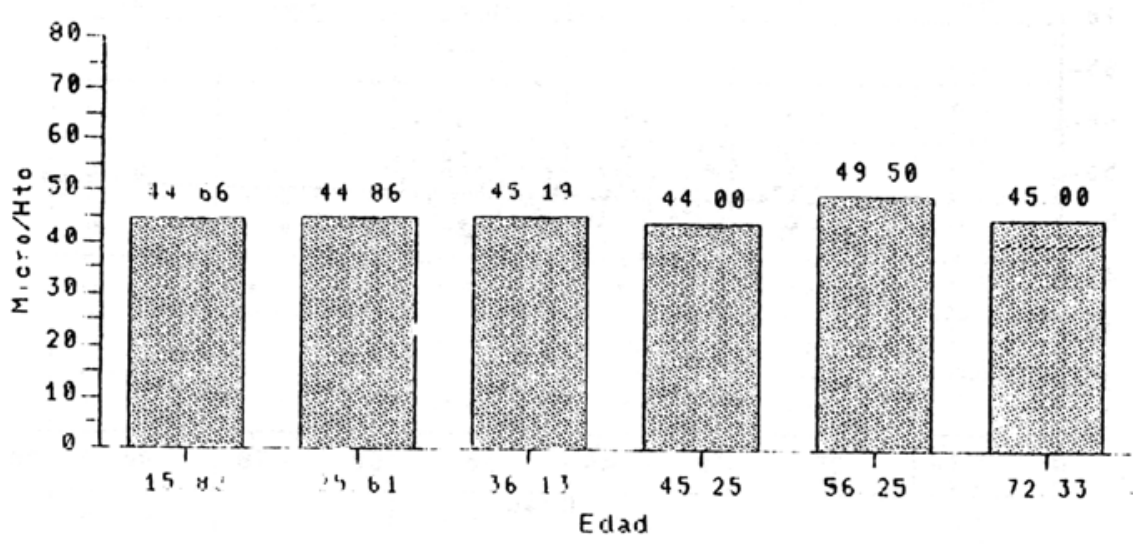

Gráfico 11. Micro-Hto en 62 mujeres nativas sanas residentes de Isluga (3650-4100 m) promedios según grupos de edad.

\section{Comentario}

Desde los primeros estudios sobre la fisiología de la adaptación a la altura en recién llegados o en residentes no nativos, siempre imperfecta comparada con la aclimatación natural de los nativos andinos, se sospechó y después confirmó la importancia de los procesos tisulares y celulares que están presentes en estos últimos. El alto contenido de mioglobina muscular en perros nativos, descubierto por Hurtado y colaboradores (1945); el incremento del número de capilares por $\mathrm{mm}^{2}$ de músculo (Hurtado et al. 1973); la menor producción de lactato y piruvato durante ejercicio máximo en nativos de Morococha respecto de nativos del mar, cada uno en su hábitat (Hurtado et al. 1956) e innumerables testimonios metabólicos, estructurales y endocrinos, apuntan a centrar en este nivel la fundamental diferencia entre la aclimatación natural y la adquirida o la adaptación aguda. Así lo puntualiza Hurtado (1964):

"es posible que la mayor eficiencia de la aclimatación natural comparada con la adquirida, dependa en uno de sus aspectos más importantes, de la presencia o mayor grado de desarrollo de procesos adaptativos tisulares en hombres y animales nacidos y desarrollados en un ambiente de altura".

Sin embargo, la facilidad de su estudio, la escasez de investigaciones holísticas orgánicas en los sujetos evaluados y lo conspicuo de las diferencias entre los parámetros de altura y de nivel del mar, en el eje de la gradiente de $\mathrm{pO}_{2}$ (de tráquea a sangre arterial y venosa), han subrayado estos valores como 


\begin{tabular}{|c|c|c|c|c|c|}
\hline \multirow{2}{*}{ Edad (años) } & \multirow{2}{*}{$\mathbf{N}^{\circ}$ de casos } & \multirow{2}{*}{ Edad media } & \multicolumn{3}{|c|}{ Hematocrito } \\
\hline & & & Media & D.D. & Rango \\
\hline $11-15$ & 6 & 13.16 & 41.50 & 2.97 & $\begin{array}{l}37.0 \\
44.50\end{array}$ \\
\hline $16-20$ & 13 & 18.15 & 46.50 & 2.54 & $\begin{array}{l}41.0 \\
50.00\end{array}$ \\
\hline $21-30$ & 13 & 26.15 & 47.85 & 2.60 & $\begin{array}{l}42.0 \\
52.00\end{array}$ \\
\hline $31-40$ & 17 & 35.65 & 49.94 & 2.79 & $\begin{array}{l}45.0 \\
55.00\end{array}$ \\
\hline $41-50$ & 7 & 45.57 & 49.50 & 2.16 & $\begin{array}{l}46.5 \\
53.00\end{array}$ \\
\hline $51-60$ & 4 & 56.00 & 47.75 & 2.33 & $\begin{array}{l}44.5 \\
50.00\end{array}$ \\
\hline 61 y más & 2 & 70.00 & 53.50 & 2.12 & $\begin{array}{l}52.0 \\
55.00\end{array}$ \\
\hline Total & 62 & & 47.89 & 3.62 & $\begin{array}{l}37.0 \\
55.00\end{array}$ \\
\hline
\end{tabular}

Gráfico 12. Microhematocrito en 62 hombres sanos, nativos residentes de los pueblos del bofedal de Isluga (altiplano chileno). (3650-4100 m.snm) PBm = 434 mmHg. Distribución según edad.

\begin{tabular}{|c|c|c|c|c|c|}
\hline \multirow{2}{*}{ Edad (años) } & \multirow{2}{*}{$\mathbf{N}^{\circ}$ de casos } & \multirow{2}{*}{ Edad media } & \multicolumn{3}{|c|}{ Hematocrito } \\
\hline & & & Media & D.D. & Rango \\
\hline $11-15$ & 6 & 12.83 & 44.00 & 2.79 & $\begin{array}{l}40.00 \\
48.00\end{array}$ \\
\hline $16-20$ & 11 & 17.45 & 45.00 & 3.11 & $\begin{array}{l}40.50 \\
50.00\end{array}$ \\
\hline $21-30$ & 18 & 25.61 & 44.86 & 2.98 & $\begin{array}{l}36.50 \\
49.00\end{array}$ \\
\hline $31-40$ & 16 & 36.13 & 45.19 & 3.15 & $\begin{array}{l}40.00 \\
52.50\end{array}$ \\
\hline $41-50$ & 4 & 45.25 & 44.00 & 3.81 & $\begin{array}{l}40.00 \\
49.00\end{array}$ \\
\hline $51-60$ & 4 & 56.25 & 49.50 & 3.63 & $\begin{array}{l}45.50 \\
54.00\end{array}$ \\
\hline 61 y más & 3 & 72.33 & 45.00 & 2.50 & $\begin{array}{l}42.00 \\
47.50\end{array}$ \\
\hline Total & 62 & & 45.14 & 3.19 & $\begin{array}{l}\mathbf{3 6 . 5 0} \\
\mathbf{5 4 . 0 0}\end{array}$ \\
\hline
\end{tabular}

Gráfico 13. Microhematocrito en 62 mujeres sanas, nativas residentes de los pueblos del bofedal de Isluga (altiplano chileno). (3650-4100 m.snm) PBm = 434 mmHg. Distribución según edad.

fundamentales en los procesos de equilibrio con los ambientes de altura. Entre ellos, él más reiterado es el valor de la hemoglobina y hematocrito. Sin negar el rol de otros procesos, por el solo incremento de la $\mathrm{Hb}$, algunos autores (Velásquez 1972) explican el aporte adecuado de oxígeno tisular en condiciones de gran altura $(4640 \mathrm{~m})$. Todo ello, a pesar también del rol deletéreo que su incremento tiene sobre el transporte de $\mathrm{O}_{2}$ la alta correlación entre Hto y viscosidad (Whittembury et al. 1968).

El gráfico de Hurtado y colaboradores (1945), correlacionando fatalmente altura, cantidad de $\mathrm{Hb}$ y $\mathrm{SaO}_{2}$, induce a pensar que una buena aclimatación se acompaña de una tasa de $\mathrm{Hb}$ y Hto distinta para cada altura y cuyo valor medio hasta 


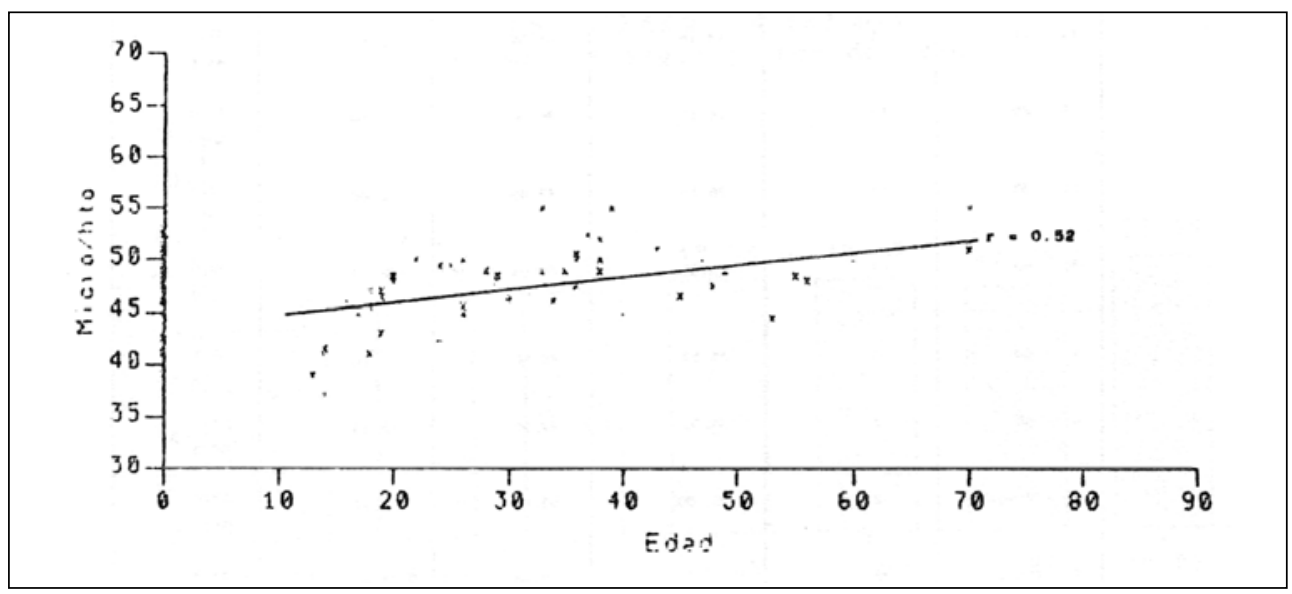

Gráfico 14. Micro-Hto en 62 hombres sanos, nativos residentes del altiplano chileno (3650-4100 m), en Isluga, Chile.

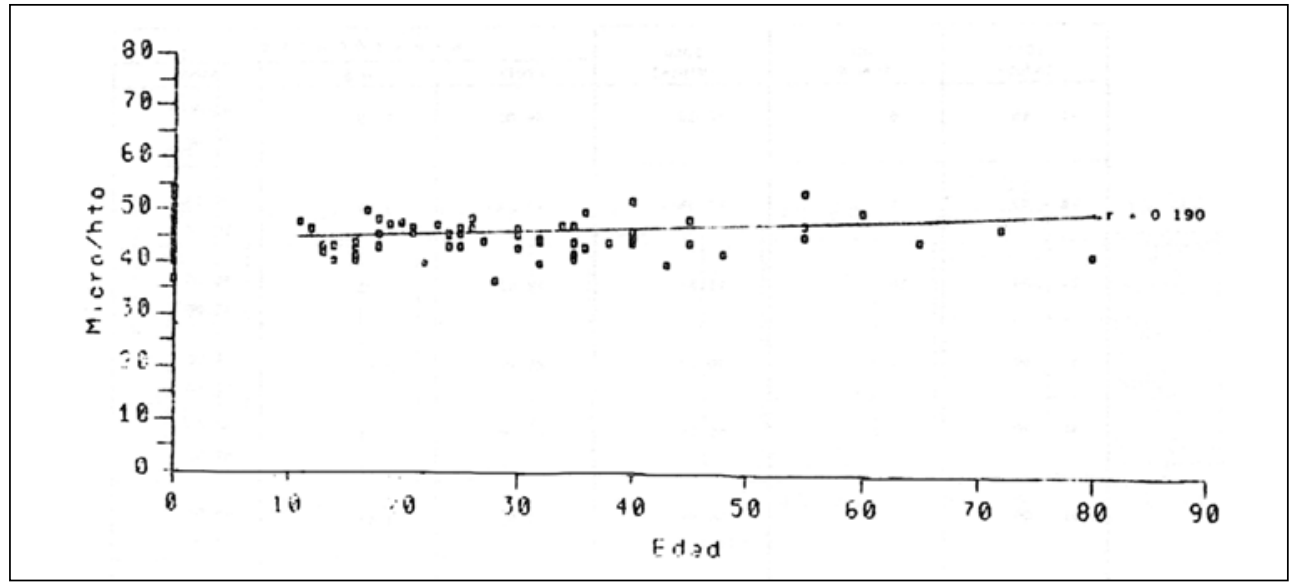

Gráfico 15. Micro-Hto en relación a edad en 62 mujeres residentes del altiplano chileno (3650-4100 m), en Isluga, Chile.

5200 m se obtiene de la curva de correlación o del cálculo de la misma (Monge y Monge 1964; Monge et al. 1965).

El principal comentario a nuestro trabajo pretende modificar este simple esquema de comprensión del complejo problema de la adaptación a la altura. En los tres grupos estudiados, la media más alta de Hto. en hombres y mujeres se presenta en la población que reside permanentemente en el nivel más bajo de altitud. La media del Hto. en 271 hombres de 21 a más de 66 años, no mineros, sanos, nativos o no del campamento minero de Chuquicamata, ubicado a $2800 \mathrm{~m}$ fue de 52.65. Sin embargo, en los nativos indígenas del altiplano de Isluga, cuya altura media es $3800 \mathrm{~m}$ y cuyas edades fluctuaron entre 11 y más de 61 años, el Hto. medio fue sólo de 47.89 y en los 21 hombres del pueblo de Socaire, a $3450 \mathrm{~m}$, todos nativos indígenas, de 49.17. Igual fenómeno ocurre en la población femenina, cuyos valores medios de Hto. fueron de 47 en 267 mujeres de Chuquicamata; 45.15 en 62 mujeres de Isluga y 45.74 en 54 mujeres de Socaire.

Por lo tanto, la correlación de la gráfica de Hurtado no es aplicable a nuestras poblaciones, debido a rasgos diferenciales entre ellas, ajenos a la sola altitud de la residencia. 


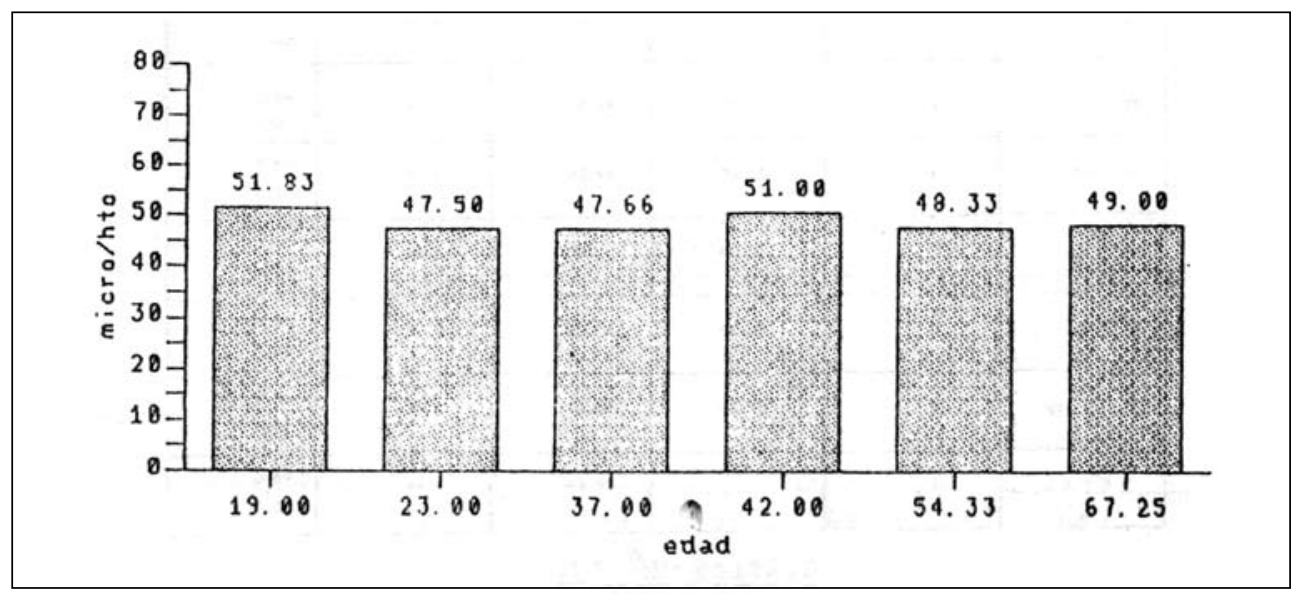

Gráfico 16. Micro-Hto en 21 hombres nativos sanos residentes de Socaire (Antofagasta) a $3450 \mathrm{~m}$. Promedios según grupos de edad.

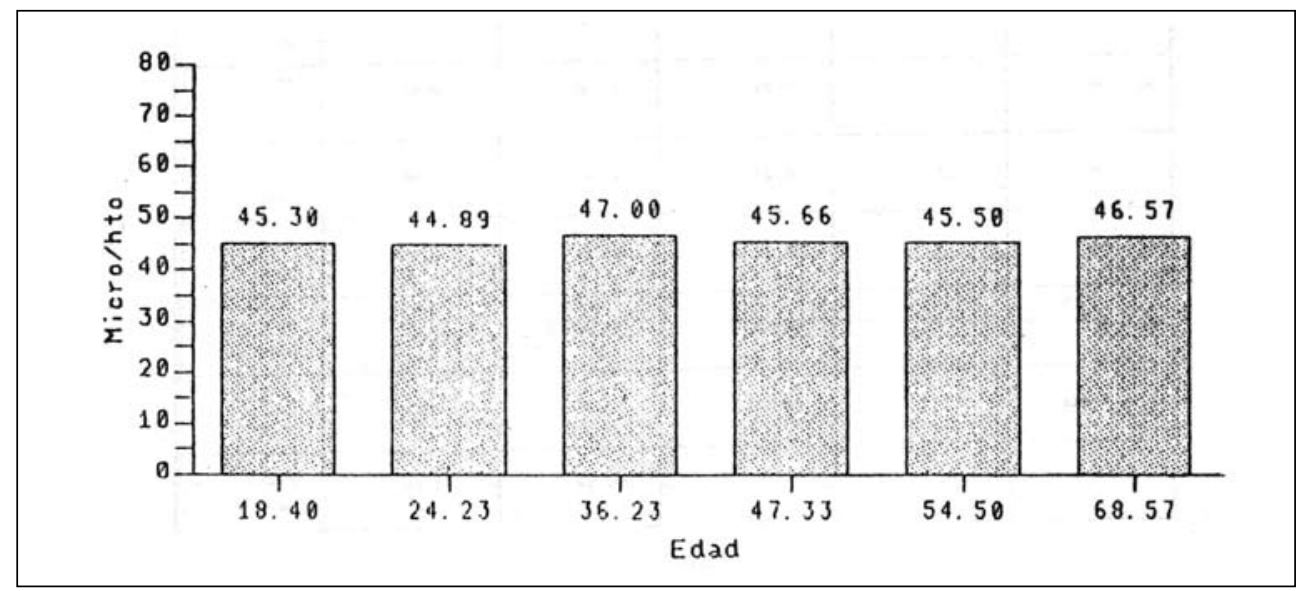

Gráfico 17. Micro-Hto en 54 mujeres nativas sanas, residentes de Socaire (Antofagasta) a $3450 \mathrm{~m}$. Promedios según grupos de edad.

El grupo de Isluga representa una población nativa altiplánica de extrema pureza genética y cultural, debida a su aislamiento. Su grado de aclimatación natural es insuperable: desconocen el mar y, en la práctica, no descendían de su nivel en el momento de la experiencia. Su modelo de vida ancestral, marcado por su carácter predominante de pastores seminómades, los mantiene en rigurosa actividad física, a menudo alcanzando cumbres por sobre los $5500 \mathrm{~m}$, con fríos rigurosos. Frugales en su alimentación, habitan una de las regiones más puras y bellas de la tierra y el grado de contaminación atmosférica y cultural del estilo occidental es mínimo. La obesidad es escasa o nula y el hábito de fumar, inexistente o escaso. El sedentarismo, solo posible en los ancianos enfermos. A esta definición del estilo de vida, que en trabajos ulteriores pretendemos describir más acabadamente, se suma la comprobación de parámetros de salud y adecuidad física que demuestran su perfecto equilibrio con el duro hábitat. Entre éstos, la capacidad aeróbica, cuyo valor de $48.6 \mathrm{ml} /$ $\mathrm{min} / \mathrm{kg}$-p, promedio en 24 hombres de 18 a 49 años, medido con ergómetro a $3800 \mathrm{~m}$, avala la perfección de su aclimatación natural.

El grupo de Socaire representa una población similar aunque con niveles de aclimatación natural menos perfectos. Su mayor sedentarismo es secundario a su 


\begin{tabular}{|c|c|c|c|c|c|}
\hline \multirow{2}{*}{ Edad (años) } & \multirow{2}{*}{$\mathbf{N}^{\circ}$ de casos } & \multirow{2}{*}{ Edad media } & \multicolumn{3}{|c|}{ Hematocrito } \\
\hline & & & Media & D.D. & Rango \\
\hline $16-20$ & 3 & 19.00 & 51.63 & 4.31 & $\begin{array}{l}48.0 \\
56.5\end{array}$ \\
\hline $21-30$ & 2 & 23.00 & 47.50 & 3.54 & $\begin{array}{l}45.0 \\
50.0\end{array}$ \\
\hline $31-40$ & 3 & 37.00 & 47.66 & 4.04 & $\begin{array}{l}44.0 \\
52.0\end{array}$ \\
\hline $41-50$ & 2 & 42.00 & 51.00 & 1.41 & $\begin{array}{l}50.0 \\
52.0\end{array}$ \\
\hline $51-60$ & 3 & 54.33 & 48.33 & 0.58 & $\begin{array}{l}48.0 \\
49.0\end{array}$ \\
\hline 61 y más & 8 & 67.25 & 49.00 & 3.07 & $\begin{array}{l}46.0 \\
53.0\end{array}$ \\
\hline Total & 21 & & 49.17 & 3.11 & $\begin{array}{l}44.0 \\
56.5\end{array}$ \\
\hline
\end{tabular}

Gráfico 18. Microhematocrito en 71 hombres sanos, nativos residentes de Socaire (Antofagasta, Chile) 3450 m.snm. PBm = 434 mmHg. Distribución según edad.

\begin{tabular}{|c|c|c|c|c|c|}
\hline \multirow{2}{*}{ Edad (años) } & \multirow{2}{*}{$\mathbf{N}^{\circ}$ de casos } & \multirow{2}{*}{ Edad media } & \multicolumn{3}{|c|}{ Hematocrito } \\
\hline & & & Media & D.D. & Rango \\
\hline $16-20$ & 5 & 18.40 & 45.30 & 3.46 & $\begin{array}{l}41.0 \\
50.0\end{array}$ \\
\hline $21-30$ & 22 & 24.23 & 44.89 & 2.80 & $\begin{array}{l}39.0 \\
50.0\end{array}$ \\
\hline $31-40$ & 13 & 36.23 & 47.00 & 3.08 & $\begin{array}{l}43.0 \\
53.0\end{array}$ \\
\hline $41-50$ & 3 & 47.33 & 45.66 & 0.58 & $\begin{array}{l}45.0 \\
46.0\end{array}$ \\
\hline $51-60$ & 4 & 54.50 & 45.50 & 3.87 & $\begin{array}{l}41.0 \\
50.0\end{array}$ \\
\hline 61 y más & 7 & 68.57 & 46.57 & 3.99 & $\begin{array}{l}42.0 \\
64.0\end{array}$ \\
\hline Total & 54 & & 45.74 & 3.10 & $\begin{array}{l}39.0 \\
54.0\end{array}$ \\
\hline
\end{tabular}

Gráfico 19. Microhematocrito en 54 mujeres sanas, nativas residentes de Socaire (Antofagasta, Chile) $3450 \mathrm{~m} . \mathrm{snm}$. PBm = $517 \mathrm{mmHg}$. Distribución según edad.

condición más agrícola que ganadera; su ubicación geográfica los lleva con frecuencia a zonas más bajas $(2400 \mathrm{~m})$. No hemos estiado aún su capacidad aeróbica. Sin embargo, los Hto son comparables a los de Isluga para su altura de residencia menor, cuya atmósfera es tan impoluta como la de las altiplanicies.

El grupo de Chuquicamata, cuyos Htos son más altos, habita un campamento minero a $2800 \mathrm{~m}$. Se exigió una residencia mínima de dos años para ser incluido en el grupo de estudio, pero la mayoría la superaba ampliamente o eran nativos. Pese a no comprender a trabajadores mineros, toda la población habita un ambiente contaminado por los humos de la industria. El estilo de vida es netamente occidental y su estándar económico social, medido con los parámetros en boga, se encuentra entre los más altos del país. El rigor climático, las facilidades de locomoción a los lugares de trabajo o recreo; la estructura del campamento y los hábitos, la convierten en una población en extremo sedentaria, lo que unido al 


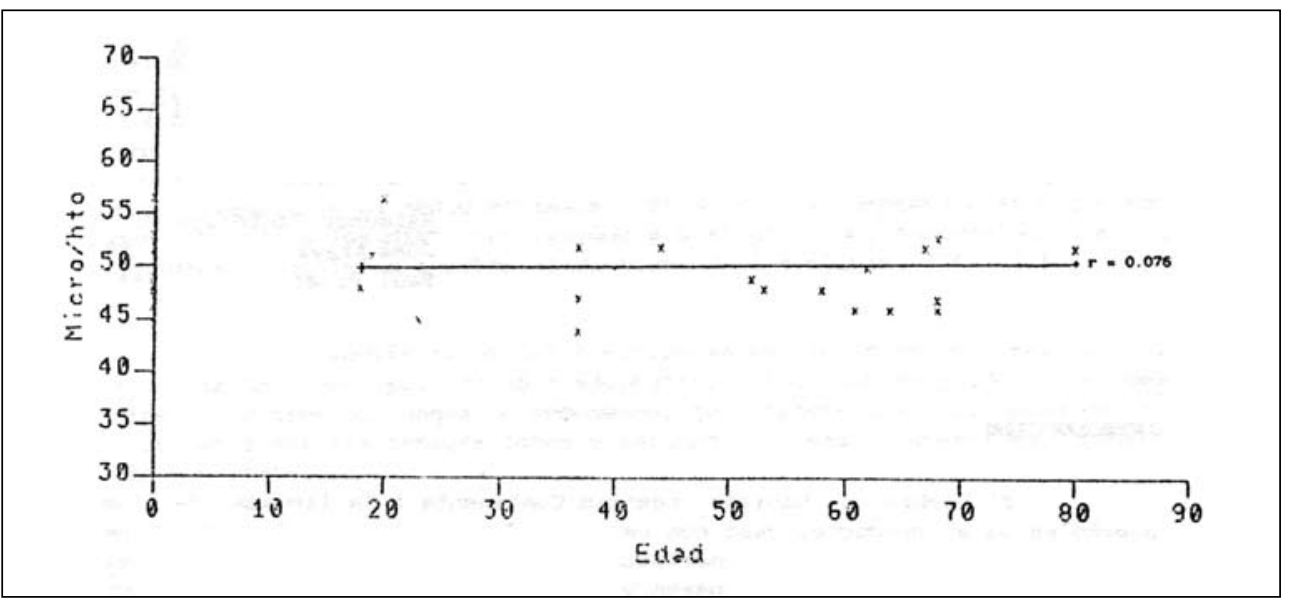

Gráfico 20. Micro-Hto en relación a edad en 21 hombres nativos sanos residentes en Socaire (Antofagasta) a 3450 m.snm.

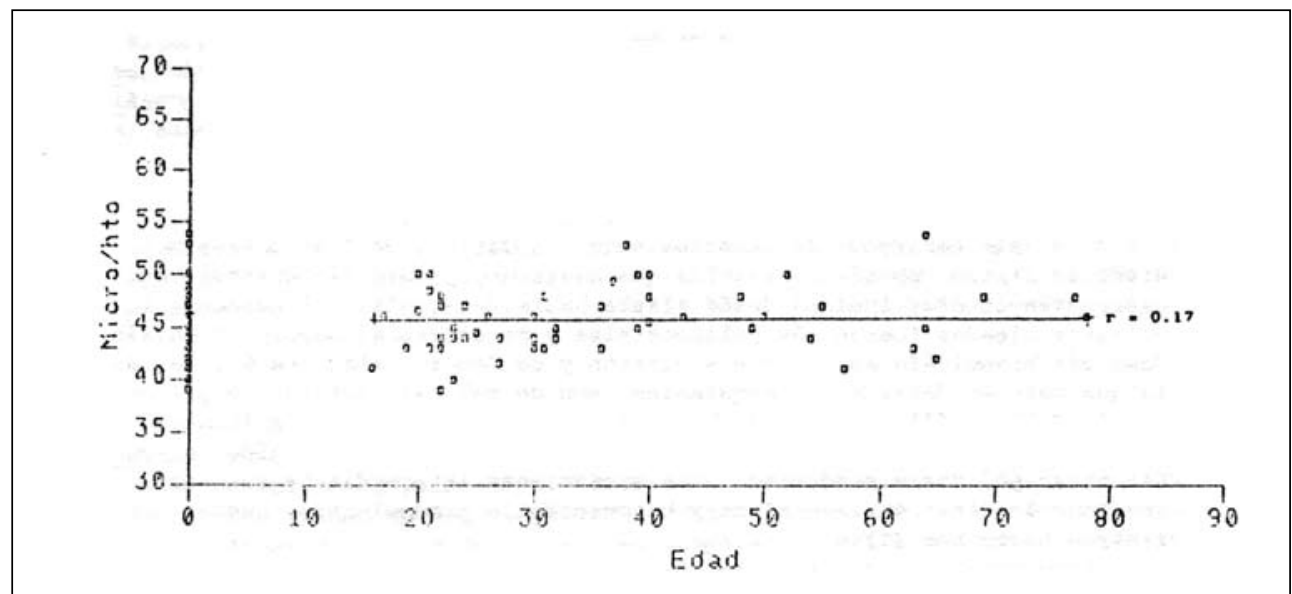

Gráfico 21. Micro-Hto en relación a edad en 54 mujeres sanas, nativas residentes en Socaire (Antofagasta) a 3450 m.snm.

tipo de alimentación, favorece una alta prevalencia de obesidad. Se trata de un grupo de gran movilidad hacia la costa y hacia las regiones bajas del centro del país. Su hábito tabáquico es frecuente. Este grupo está siendo rigurosamente estudiado pero la sola definición de su estilo de vida lo aleja del modelo ideal de aclimatación natural, descrito en las comunidades nativas de Isluga y Socaire.

Sostenemos, por tanto, que un individuo o grupo residente a una altitud dada poseerá una $\mathrm{Hb}$ y/o Hto distintos, según sea la calidad de su aclimatación y la participación en su logro, de todos los niveles del sistema de transporte de $\mathrm{O}_{2}$. A una altura dada, a peor aclimatación, mayor Hto, en ausencia claro está, de anemia. Sea por tiempo escaso de residencia o por intermitente cambio de mar a altura; sea por factores que afecten distintos órganos o sistemas (irritación bronquial, edema pulmonar intersticial, carboxihemoglobina elevada en los fumadores, menor desarrollo del lecho vascular, sedentarismo, etc.), la elevación del Hto respecto de la media de su grupo o de otros sujetos mejor aclimatados indicará que su transporte y utilización de $\mathrm{O}_{2}$ es imperfecto y requiere de un ajuste fácil pero no ideal para mejorarlo. Lo mismo ocurre al comparar poblaciones que como las nuestras se diferencian en altitud de residencia, tipo de vida y grado de aclimatación. 
El nativo andino posee desde su nacimiento y a través de su desarrollo una multiplicidad de rasgos a distintos niveles que integrados armoniosamente explican la perfección de su aclimatación natural y la alta eficiencia de su trabajo aeróbico. Ninguno es suficiente por sí solo. Ni el mayor desarrollo pulmonar ni su particular estructura y función cardiovascular, ni su mayor lecho capilar, ni su patrón neuroendocrino y ventilatorio, ni su diferente metabolismo, en especial el vinculado a los carbohidratos, ni el misterio de su bioquímica celular: todos juntos, apoyados por la sabiduría ancestral diseñada en milenios de residencia y cultura andinas (Hurtado 1964; Velásquez 1972; Santolaya 1981). Este planteamiento, sostenido por nosotros en diversos foros (Santolaya 1975, 1981), es avalado por hallazgos de otros autores. Roy (1964-72) halla Htos de 45.2 \pm 3.3 en ladakhi scouts residentes a $3600 \mathrm{~m}$, idénticos a los $45.0 \pm 4.2$ de residentes hindúes a nivel del mar. Garrutto (1976) encuentra Hto de 51.4 a 4000 m, en Nuñoa (Perú). Xie Cheng-Fan y Pei Shu-Xuan (1980) encuentran diferencias en una misma altitud en el recuento de eritrocitos entre campesinos y pastores nativos (4.91) respecto de trabajadores nativos no rurales (5.41) en el área de Lhasa, a $3658 \mathrm{~m}$.

El segundo comentario que se deduce de nuestros datos es la ausencia de correlación entre la edad y el Hto en nuestras poblaciones de altura, a diferencia de lo publicado por Monge y Whittembury (1972) y Sime y colaboradores (1975). El hallazgo de Monge de $\mathrm{r}=0.693$ para 11 hombres de Puno $(3800 \mathrm{~m})$; 0.747 para 26 de Cerro de Pasco (4200 m) y 30 de Morococha (4500 m), contrasta con nuestras $\mathrm{r}$ de 0.076 en 21 hombres de Socaire $(3450 \mathrm{~m}) ; 0.52$ en 62 hombres de Isluga (altura media $3800 \mathrm{~m}$ ) v 0.089 en 271 hombres de Chuquicamata (2800 m). Hemos discutido estas cifras con los autores mencionados, concordando en que parte de la diferencia es debida a la distinta edad de la población estudiada y a que esa correlación probablemente es más evidente en altitudes mayores.

Creemos que la investigación debe extenderse y que la definición de las características de cada población de altura estudiadas debe afinarse en pos de un análisis más certero del concepto de aclimatación normal y patológica. Entre ellas, el estudio del estilo de vida tradicional de las poblaciones andinas y su ejemplo para los nuevos asentamientos de altura debe ser prioritario.

\section{Resumen y conclusiones}

1. Se presentan los resultados obtenidos en 737 hematocritos de residentes permanentes de alturas diversas, de ambos sexos, de asentamientos ubicados en los Andes del norte de Chile (regiones de Tarapacá y Antofagasta).

2. El valor del Hto en 271 hombres sanos, residentes, nativos o no del campamento cuprífero de Chuquicamata, no minero, altitud $2800 \mathrm{~m}$ fue

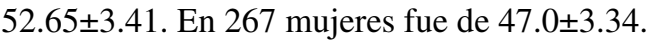

3. En 62 hombres, nativos indígenas, residentes de los pueblos del altiplano chileno de Tarapacá, de la ribera del bofedal de Isluga, altitud entre 4100 y $3650 \mathrm{~m}$ el Hto fue de $47.89 \pm 3.62$ y en 62 mujeres, de $45.14 \pm 3.19$.

4. En 21 hombres nativos indígenas residentes del pueblo atacameño de Socaire (Antofagasta), ubicado a $3450 \mathrm{~m}$, el Hto fue de $49.17 \pm 3.11$ y en 54 mujeres, de 45.74+3.10.

5. Se enfatiza la ausencia de relación en los tres grupos estudiados, entre Hto y nivel de altitud, como le afirma la literatura.

6. Se analizan las diferencias de estilo de vida entre los grupos estudiados y del tipo de hábitat como posibles explicaciones de las cifras de Hto obtenidas. Los grupos de Socaire e Isluga están constituidos por nativos indígenas, residentes permanentes de altura, en un hábitat no urbano, cuyo estilo de vida corresponde al andino ancestral. El de Chuquicamata, heterogéneo desde el punto de vista racial y del lugar de nacimiento, representa el tipo de vida habitual de un campamento minero andino, de estilo occidental, donde la contaminación atmosférica, la obesidad, el sedentarismo, el hábito de fumar y los viajes a la costa son frecuentes.

7. No se encontró evidencia de correlación en ninguno de los tres grupos estudiados entre edad y Hto.

8. Se reitera la necesidad de estudiar holísticamente diversas poblaciones residentes de distintas alturas, para evaluar el rol del estilo de vida en la calidad de la aclimatación a diversos niveles de altitud. 


\section{REFERENCIAS CITADAS}

BARCROFT, J., 1914. The respiratory functions of the blood ( $1^{\mathrm{a}} \mathrm{Ed}$.). Cambridge, Londres.

1925. The respiratory functions of the blood. Cambridge University Press.

COMROE, H. J., 1965. Physiology of respiration. The Year Book Medical Publication Inc., Chicago.

COMROE, H. J. y otros, 1955. The lung, The Year Book Medical Publication Inc., Chicago.

DEJOURS, P., 1962. Physiologie de la respiration. Flammarion Medicine Sciences.

DILL, D. B., H. T. EDWARDS, S. A. FOLLING, A. M. OBERG, PAPPENHEIMER, Jr. y J. H. TALBOTT, 1931. Adaptations of the organism to changes in oxygen pressure. American Journal of Physiology 71: 47-63.

DILL, D. B., E. H. CHRISTENSEN y H. T. EDWARDS, 1936. Gas equilibria in the lungs at high altitudes. American Journal of Physiology 115: 530-538.

DONOSO, H., E. APUD, M. C. SAÑUDO y R. SANTOLAYA, 1971. Capacidad aeróbica como índice de adecuidad física en muestras de poblaciones (urbanas y nativas de la altura) y en un grupo de atletas de selección. Revista Médica de Chile 99: 719-731.

DONOSO, H., B. R. SANTOLAYA y E. APUD, 1976. Hematocrit in native population living permanently at high altitude in the Andean plateau of Northern Chile. Les Colloques de l'Institut de la Santé et de la Recherche Medicale 63: 547-552.

FENN, O. W. y H. RAHN, 1964. Handbook of physiology; Section 3: Respiration, vol. 1, p. 770. American Physiology Society, Washington D. C.

GARRUTTO, R., 1976. Hematology. En Man in the Andes. A multidisciplinary study of high altitude quechua, P. T. Baker y M. A. Little (Eds.) Hutchinson and Ross, Inc. Publ., Dowden.

HURTADO, A., 1964. Animals in high altitude: Resident man. Handbook of Physiology. Environment, cap. 54, pp. 843860.

HURTADO, A. y otros, 1945. Influence of anoxemia on the hemopoietic activity. Arch. Int. Med. Med. 75: 284-323.

1956. Mechanisms of natural acclimatization: Studies on the native resident of Morococha, Perú, at an altitude of 14900 feet. Report 56-1, University School of Aviation Medicine, Randolph Field, Texas, pp. 1-62.

- Blood gas transport and acid-base balance at sea level and at high altitude. Report 56-104, Air University School of Aviation Medicine, Randolph Field, Texas.
HURTADO, A., A. ROTTA, C. MERINO y J. PONS, 1973. Studies of myohemoglobin at high altitudes. American Journal of Medical Science 194: 708-713.

KEYS, A., 1936. The physiology of life at high altitudes. Scientific Monthly 43: 289-312.

KEYS, A., HALL y BARRON, 1936. American Journal of Physiology 115: 292.

MAYORGA, M., 1978. Contribución al estudio de la influencia de la presión atmosférica sobre el organismo. Archivos de Biología Andina UNMSM 8 (1-4).

Mc CONN R. y J. B. DERRICK, 1972. The respiratory function of blood: Transfusion and blood storage. Anesthesiology 36: 119-127.

MERINO, C., 1950. Studies on blood formation and destruction in the polycytemia of high altitude. Blood 5: 1-3.

MONGE, C., 1929. Les erythremies de l'altitude. Editorial Masson, París.

MONGE, C. y C. MONGE, 1964. Mal de montañas crónico. Actas del $8^{\circ}$ Congreso Internacional de Medicina Interna. Buenos Aires.

MONGE, C. y J. WHITTEMBURY, 1972. High altitude, haematocrit and age. Nature 238 (5362): 278-279.

MONGE, C. y otros, 1954. Aclimatación en los Andes: Forma y función del tórax. Perú Indígena 5 (13): 3-15.

MONGE, C., R. LOZANO y J. WHITTEMBURY, 1965. Nature 207: 770

REYNAFARJE, C., 1957. The influence of high altitude on erythropoietic activity. Homeostatic mechanisms. Brookhaven Symposia in Biology 10: 132-146.

ROSSIER, P. R. y otros, 1962. Physiologie et physiopathologie de la respiration. Ed. Delachaux et Niestlé, Neuchatel.

ROTTA, A. y otros, 1956. Pulmonary circulation at sea level and at high altitude. Journal of Applied Physiology 9: 328-336.

ROY, B. S., 1964-2. Circulatory and ventilatory effects of high altitude acclimatization and de acclimatization of indian soldiers. A prospective study. Indian Council of Medical Research, Nueva Delhi.

SANTOLAYA, R., 1975Ms. Conferencia Adaptación y aclimatación a la altura. Universidad Peruana Cayetano Heredia.

1981Ms. Parámetros de aclimatación a la altura: Poblaciones del campamento de Chuquicamata (2850 m, Desierto de Atacama), altiplano y precordillera chilena de Tarapacá y Antofagasta. Trabajo presentado al International Symposium on acclimatization, adaptation and tolerance to high altitudes National Institutes of Health; Fogarty International Center (1981) Bethesda. 
1981. Adaptación y aclimatación a la altura: Un proceso integral. Revista Médica del Hospital Roy H. Glover 1: 51-60.

SANTOLAYA, R. y H. DONOSO, 1976. Estudio de la prevalencia de infección TBC, según la técnica de la tuberculina standard TR 23 con Tween 80 de 2 UT, intradérmica, en poblaciones nativas residentes de la precordillera y altiplano chilenos. Les Colloques de l'Institut de la Santé et de la Recherche Medicale 63.

SANTOLAYA, R., H. DONOSO, E. APUD y M. C. SAÑUDO, 1973. Electrocardiograma y capacidad aeróbica en nativos residentes de altura del altiplano chileno, como índice de aclimatación. Revista Médica de Chile 101: 433.

SANTOLAYA, R. y otros, 1981. Hematocrito, hemoglobina y presión de $\mathrm{O}_{2}$ arterial en 270 hombres y 266 mujeres sanas residentes de altura (2800 m). Revista Médica del Hospital Roy H. Glover 1: 17.

SANTOLAYA, R., J. ARAYA, A. VECCHIOLA, H. FABRES, R. PRIETO y R. VERGARA, 1982. Gases y $\mathrm{pH}$ en sangre arterial en 176 hombres y 162 mujeres sanas, trabajadoras no mineras residentes de altura $(2800 \mathrm{~m})$. Revista Médica del Hospital Roy H. Glover 2 (2).

SANTOLAYA, R., J. ARAYA y R. PRIETO, 1983. Aclimatación al medio de altura: Un proceso integral. Versiones abreviadas. Primer Encuentro Científico sobre el Medioambiente Chileno vol. 2, pp. 1-5, La Serena.

1983Ms. Capacidad vital, talla, peso y superficie corporal en dos poblaciones adultas, sanas, residentes de altura del
Norte Grande de Chile: 98 nativos del bofedal de Isluga (altura promedio $3800 \mathrm{~m}$ ) y 351 residentes del campamento cuprífero de Chuquicamata (2800 m). Ponencia presentada al Simposio de Arqueología Atacameña, San Pedro de Atacama (Chile), este volumen.

SIME, F., C. MONGE y J. WHITTEMBURY, 1975. Age as a cause of chronic mountainin sickness (Monge's disease). International Journal of Biometeory 19 (2): 93-98.

VALDIVIA, E., 1956. Mechanisms of natural acclimatization. Capillary studies at high altitudes. Rept. 55-101, School of Aviation Medicine, USAF, Randolph Field, Texas.

VELASQUEZ, M. T., 1972. Análisis de la función respiratoria en la adaptación a la altitud. Tesis de Doctorado, Lima.

VIAULT, E., 1891. Sur la quantité d'oxygene contenue dans le sang des animaux des hauts plateaux de l'Amérique du Sud. Compt. Rend. Academie Scientifique 112: 295-298.

WEST, B. J., 1975. Respiratory physiology. The Essentials. The Williams and Wilkins Co., Baltimore.

WHITTEMBURY, J., R. LOZANO, C. TORRES y C. MONGE, 1968. Blood viscosity in high altitude polycythemia. Acta Fisiológica Latinoamericana 18: 355-359.

XIE CHENG-FAN, PEI SHU-XUAN, 1980. Some physiological data of sejourners and native highlanders at three different altitudes in Xizang. VII Environmenta 1980 Ecology. Science Press, Beijing. Gordon and Breuen, Science Publishing Inc. Nueva York. 